\title{
Binary nature and elemental abundances of 2 Lyn and HD 169981^
}

\author{
H. Lehmann ${ }^{1}$, I. Egorova ${ }^{2,3}$, G. Scholz ${ }^{4}$, G. Hildebrandt ${ }^{4}$, and S. M. Andrievsky ${ }^{2,3}$ \\ 1 Thüringer Landessternwarte Tautenburg, Karl-Schwarzschild-Observatorium, 07778 Tautenburg, Germany \\ 2 Department of Astronomy, Odessa State University, Shevchenko Park, 270014 Odessa, Ukraine \\ 3 Isaac Newton Institute of Chile, Odessa Branch \\ e-mail: egorova@deneb.odessa.ua, sergei@deneb.odessa.ua \\ 4 Astrophysikalisches Institut Potsdam, An der Sternwarte 16, 14483 Potsdam, Germany \\ e-mail: ghildebrandt@aip.de, gscholz@aip.de
}

Received 6 December 2002 / Accepted 27 January 2003

\begin{abstract}
We analyse high-resolution spectrograms of the early A-type stars HD 169981 and 2 Lyn. For the spectroscopic and photometric binary HD 169981 we determine the abundances of certain chemical elements. The derived atmospheric parameters show the star to be an A giant. The abundances of the elements $\mathrm{Mg}, \mathrm{Si}, \mathrm{Ti}, \mathrm{Cr}$, and $\mathrm{Fe}$ are very close to solar, only $\mathrm{C}$ and $\mathrm{O}$ show slight underabundances. 2 Lyn has been primarily studied for the occurrence of radial velocity variations. The star is probably a spectroscopic binary with a period of about 3.6 years. Preliminary orbital elements are derived. The search for shorter radial velocity variations gives hints of periods of 69.2, 1.56 and 1.53 days. Both of the last-mentioned periods are in the order of the expected rotation period of the star. The stellar parameters are estimated and for certain elements we determined the abundances.
\end{abstract}

Key words. stars: binaries: spectroscopic - stars: abundances - stars: oscillations - stars: individual: 2 Lyn stars: individual: HD 169981

\section{Introduction}

HD 169981 and 2 Lyn are early A-type stars recently studied by photometric and spectroscopic observations. According to these investigations HD 169981 is very probably not only a spectroscopic but also an eclipsing binary and 2 Lyn seems to be a spectroscopic binary and to show pulsations. We check these results on the basis of time series of high-resolution spectra.

HD 169981. HD 169981 (HR 6917, GC 25165, Boss 4669, HIP 90342) has been known for many decades to be a spectroscopic binary (Young 1919). Recently Lehmann et al. (2001, hereafter Paper I) carried out a closer investigation of the binary nature of the star. On the basis of 384 CCD spectrograms the authors derived more precise orbital elements. A search for periods in the residuals of the orbital solution gave no periodicity exceeding a radial velocity (hereafter RV) amplitude of about $250 \mathrm{~m} \mathrm{~s}^{-1}$. According to both our photometry obtained in 1999 and 2000 and the Hipparcos data there is strong evidence that HD 169981 is an eclipsing binary. The mass function and

Send offprint requests to: $\mathrm{H}$. Lehmann,

e-mail: lehm@tls-tautenburg.de

* The research is based on spectroscopic observations made with the 2-m telescope at the Thüringer Landessternwarte Tautenburg, Germany. the shape of the photometric curve indicate a binary consisting of an early A giant primary and a mid $\mathrm{K}$ main-sequence secondary. Thus, we expect no measurable contribution of the secondary at the wavelengths covered by our spectra.

In order to complete the results and to corroborate our assumptions on the binary model, we use our spectrograms also to derive the atmospheric parameters and the elemental abundances of HD 169981.

2 Lyn. The A2 V star 2 Lyn (HD 43378, HR 2238, GC 8068, HIP 30060) was recently studied by Caliskan \& Adelman (1997, hereinafter C\&A) for elemental abundances. The derived abundances of 14 chemical elements are very close to solar. The authors concluded from the observed RV variations that the star might be a spectroscopic binary.

2 Lyn belongs to a group of late B- and early A-type stars, which were investigated by Scholz et al. (1998, hereinafter Paper II) for the occurrence of short-term RV and light variations. The RV study included 20 photographic spectrograms obtained with the coudé spectrograph of the 2-m telescope of the Thüringer Landessternwarte Tautenburg (hereinafter TLS) and the $17 \mathrm{RVs}$ given by C\&A. Although the analysis yielded hints of a binary nature of 2 Lyn we could not establish a unique orbital solution because of the poor time sampling of the data. Concerning the existence of short-term RV variations, the small number of existing spectrograms and their accuracy did not 
allow us to draw any conclusions. We are sure, however, that the $\delta$ Scu-like RV variations of several tens of $\mathrm{km} \mathrm{s}^{-1}$ found by Antonello et al. (1978) for the hydrogen lines are not present in our spectrograms. This fact, as well as the solar-like abundances of 2 Lyn found by C\&A, raises doubt on Antonello's et al. statement that 2 Lyn could be a cryptic Am star showing $\delta$ Scu pulsations.

In Paper II we also analysed the Hipparcos photometry data of $2 \mathrm{Lyn}$. The period search within the 83 values gave a hint of a period of $0.237 \mathrm{~d}$ with a semi-amplitude of $3 \mathrm{mmag}$. Although the false-alarm-probability of this period does not exceed $1 \%$, the rms of the residuals is of the same order as the found amplitude.

To set more stringent limits on 2 Lyn's binary nature and to look for evidence of other RV variability we applied additional spectroscopic observations. The newly obtained time series of high-resolution spectra allow us to derive further a more accurate value of the projected rotational velocity, necessary to estimate the stellar parameters of the star. For $v \sin i$ we find very different values in the literature: Uesugi \& Fukuda (1982), $10 \mathrm{~km} \mathrm{~s}^{-1}$; Abt \& Morrell (1995), $35 \mathrm{~km} \mathrm{~s}^{-1}$; Solano \& Fernley (1997), $60 \mathrm{~km} \mathrm{~s}^{-1}$.

\section{Observations and reductions}

\subsection{HD 169981}

To determine the atmospheric parameters and the elemental abundances of HD 169981 we used one well-exposed spectrogram of our observational set noted in Paper I. The two-pixel resolution of these spectra is 37000 , all other parameters are comparable to the parameters described below for the spectra of 2 Lyn.

\subsection{Lyn}

Observations. The RV study of 2 Lyn is based on 901 highresolution echelle spectra obtained over 27 nights during JD 2450917 to 2452428 . All spectra were taken with the CCD-echelle-spectrograph at the Coude focus of the 2-m telescope of the Thüringer Landessternwarte in Tautenburg. Spectra cover a wavelength range from 470 to $740 \mathrm{~nm}$ with a spectral resolution of 60000 and a typical $S / N$ of 200 . The exposure time was $300 \mathrm{~s}$ with a mean time sampling of about $400 \mathrm{~s}$. In the calculation of the orbital solution we included also older photographic spectra obtained at the Rozhen observatory in Bulgaria and photographic spectra taken at the TLS as well as the RVs published by C\&A. All data are taken as HJD and summarized in Table 1.

Data reduction. The Tautenburg echelle spectra were reduced using MIDAS standard reduction packages. Reduction included filtering of cosmics, background subtraction, flat fielding, extraction of spectra, wavelength calibration by $\mathrm{Th}-\mathrm{Ar}$ spectra, and normalization to the local continuum. Spectra were corrected for instrumental wavelength shifts using a large number of telluric lines of $\mathrm{O}_{2}$. The internal accuracy of the wavelength calibration corresponds to a rms of better than $60 \mathrm{~m} \mathrm{~s}^{-1}$. The mean accuracy of the telluric corrections was $45 \mathrm{~m} \mathrm{~s}^{-1}$.
Table 1. Journal of the spectroscopic observations of 2 Lyn. $N_{\mathrm{n}}$ denotes the total number of obtained spectrograms and $N_{\mathrm{r}}$ the number of the runs.

\begin{tabular}{llrr}
\hline \hline source & JD 2 40000+ & $N_{\mathrm{n}}$ & $N_{\mathrm{r}}$ \\
\hline C\&A & $47831-49617$ & 17 & 8 \\
TLS & 48605 & 18 & 1 \\
Rozheto & $49826-50001$ & 6 & 2 \\
TLS $_{\text {ccd }}$ & $50917-52428$ & 901 & 27 \\
\hline
\end{tabular}

Measurement of the radial velocity. Radial velocities were measured first for a number of metallic lines using Gaussian fits of line profiles. Because of the high rotational line broadening (see Sect. 4), and since we did not observe systematic differences for different ions or multiplets, we decided to measure the RVs from a cross-correlation of the spectra with a template where we obtained better accuracy. For the cross-correlation we used the spectral range from 470 to $740 \mathrm{~nm}$ excluding the Balmer lines and the wavelength intervals with larger telluric contributions. The template was built up iteratively starting with an arbitrary spectrum of $2 \mathrm{Lyn}$, and then from the mean of all spectra shifted for the individual RVs obtained in the previous step. The mean internal accuracy of the cross-correlation was $35 \mathrm{~m} \mathrm{~s}^{-1}$. Together with the errors of wavelength calibration and telluric corrections given above we can estimate a lower limit for the mean errors of the finally obtained RVs of $85 \mathrm{~m} \mathrm{~s}^{-1}$.

\section{Radial velocities of $\mathbf{2}$ Lyn}

\subsection{Approximate orbital solution}

For the determination of the orbital elements we use a computer code based on the method of differential corrections (Schlesinger 1908) which determines the orbital elements and the orbital period simultaneously.

Figure 1 shows the temporal behaviour of the obtained TLS (CCD) RVs of $2 \mathrm{Lyn}$, together with the older, mainly photographic, measurements. Since it was not possible to compute an unique orbital solution from our new RVs alone (the calculation does not converge because of the poor phase coverage), we included also the older RVs of much lower accuracy into the calculations.

First we computed a common solution from all data and without any weighting. The solid curve in Fig. 1a shows the unweighted solution. Considering the position of the data, two of C\&A's RVs are more or less outliers (marked by the numbers 1 and 2). They were omitted from the orbital calculations. The resulting orbital elements are listed in Table 2 as solution I.

In the next step we used a moderate weighting scheme for the different data sets. According to that, the TLS (CCD) spectra have the weight 10 and all other the weight 1 . The result is listed in Table 2 as solution II and shown in Fig. $1 b$.

Finally, we weight the data according to their accuracy. C\&A estimated a mean error of their RVs of about $1 \mathrm{~km} \mathrm{~s}^{-1}$. According to the accuracy of our data of better than $100 \mathrm{~m} \mathrm{~s}^{-1}$ we give our RVs a weight of 100 and C\&A's data a weight of 1 . 
Table 2. Orbital elements derived from all data using different weighting schemes. Solution I: unweighted data. Solution II: TLS (CCD) spectra have weight 10 , all other weight 1 . Solution III: TLS (CCD) spectra have weight 100 , all other weight $1 . T$ is the epoch of periastron passage, given as $T+2450000$. Errors of the elements are given in parentheses in units of the last two digits. $\mathrm{rms}_{\mathrm{TLS}}$ gives the rms of the residuals if we apply the common solution obtained from all data only to the TLS (CCD) RVs.

\begin{tabular}{lrrrl}
\hline \hline & solution I & solution II & solution III & \\
\hline$P$ & $1333.5(2.3)$ & $1355.8(4.9)$ & $1374.5(8.1)$ & $\mathrm{d}$ \\
$K$ & $9.36(3.5)$ & $3.11(16)$ & $6.2(11.1)$ & $\mathrm{km} \mathrm{s}^{-1}$ \\
$e$ & $0.642(95)$ & $0.529(41)$ & $0.78(34)$ & \\
$T$ & $690(3.9)$ & $847.9(6.1)$ & $834.0(8.1)$ & \\
$\omega$ & $160.0(3.7)$ & $161.5(2.9)$ & $157(15)$ & $\mathrm{deg}$ \\
$\gamma_{\text {TLSccd }}$ & $-4.32(43)$ & $-2.257(56)$ & $-2.24(44)$ & $\mathrm{km} \mathrm{s}^{-1}$ \\
$\gamma_{\text {TLSphoto }}$ & -3.3 & -1.0 & -1.1 & $\mathrm{~km} \mathrm{~s}^{-1}$ \\
$\gamma_{\text {Rozhen }}$ & -3.7 & -1.7 & -1.7 & $\mathrm{~km} \mathrm{~s}^{-1}$ \\
$\gamma_{\mathrm{C} \& \mathrm{~A}}$ & -4.3 & -2.8 & -3.2 & $\mathrm{~km} \mathrm{~s}^{-1}$ \\
$\mathrm{rms}_{\mathrm{TLS}}$ & 0.372 & 0.364 & 0.363 & $\mathrm{~km} \mathrm{~s}^{-1}$ \\
\hline & & & &
\end{tabular}

The Rozhen RVs as well as the RVs from the photographic TLS spectra practically represent single points in the orbital phase diagram. Since we allow for different $\gamma$-velocities for data sets of different origin, these points are shifted during the orbit calculation to a suitable position and have no remarkable influence on the derived solution. We give these data also a weight of 1 . The derived elements are listed in Table 2 as solution III, Fig. 1c shows the corresponding orbital curve.

From Table 2 and Fig. 1 we can conclude the following:

(1) The errors of the elements and the rms of the residuals of the TLS (CCD) RVs are not or are only marginal reduced by giving our RVs of higher accuracy a higher weight. The reason for this is that the RVs include a remarkable contribution of additional intrinsic variations.

(2) By giving our new RVs a higher weight, four of C\&A's RVs (marked in Fig. 1 by numbers 1 to 4 ) are clear outliers. Thus, the reliability of C\&A's RVs is in doubt and we will use them only to get a first solution as a starting point. We will see in the next section that we get stable solutions from the new data alone as long as we take at least one additional sinusoidal variation into account.

(3) Although the rms of solutions I to III are almost equal, the obtained orbital elements strongly depend on the applied weighting scheme. Tests also showed that the addition of some assumed sinusoidal variations strongly modifies the orbital elements. Thus, any search for further intrinsic periodicities should also include a simultaneous optimization of the orbital elements.

\subsection{Period search and cleaned orbital solution}

In the following we search for the intrinsic RV variations mentioned above. To get a consistent solution that includes the orbital as well as the (possible) additional short-term variations we extended our computer code that determines the orbital
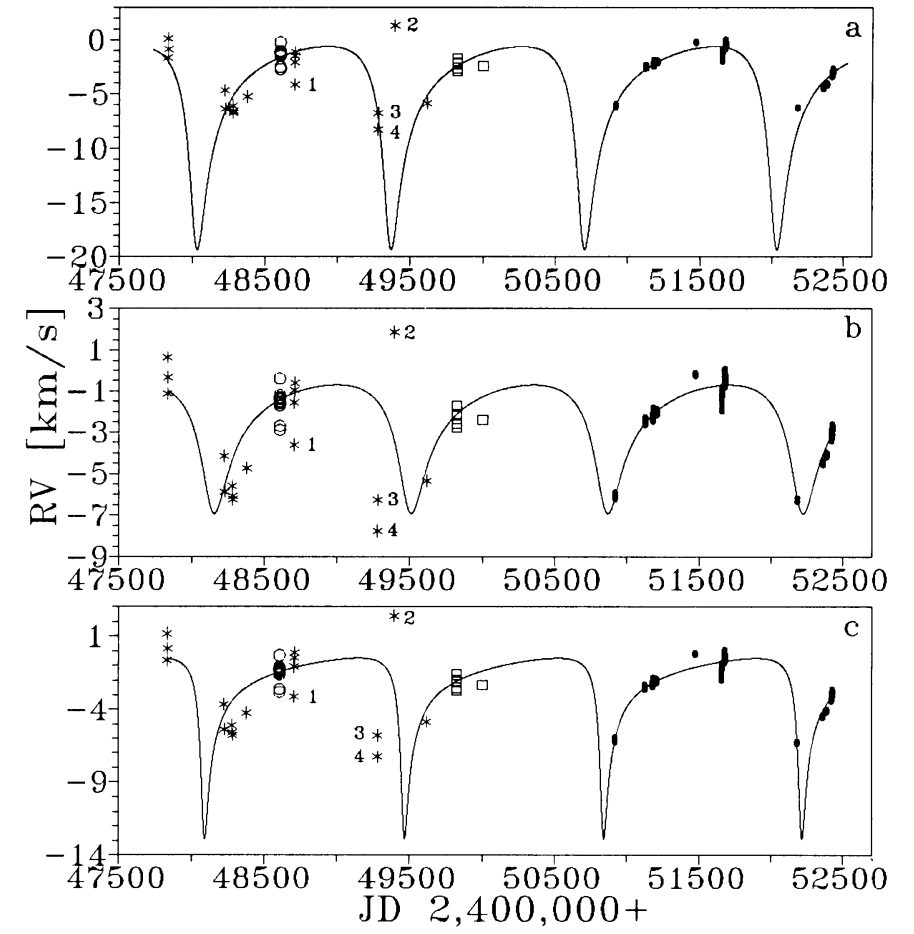

Fig. 1. Radial velocities of 2 Lyn based on TLS CCD spectra (dots), on Rozhen spectra (open squares), and on the TLS photographic spectra (open circles), as well as the RVs published by C\&A (asterisks). Four of C\&A's RVs are marked by numbers (see text). The solid curves in panels a) to c) show orbital solution I to III, respectively. The $\gamma$ corrections following from each solution were already applied to the data.

period and elements (see Sect. 3.1). Starting with given values for the orbital parameters, the code optimizes the orbital period and elements as well as the amplitudes and phases of an arbitrary number of sinewaves. It is not possible in this way to derive also the unknown periods of the sinewaves, however. The reason is that the frequency spectrum of the sinewave contributions is dense compared to that of the orbital period. We built periodograms where the abscissa is the period or frequency of the sinewave contribution in question and the ordinate is

$S=1-\sigma / \sigma_{0}$,

where $\sigma$ is the variance of the residuals after applying the actual fit and $\sigma_{0}$ is the variance of the best fit not including the sinewave that we want to determine but all other contributions determined so far. For any value of the abscissa, a complete set of starting parameters for the orbit and the fixed periods of all sinewave contributions are given to the program which calculates than the optimized orbital period and elements, and the amplitudes and phases of the sinewaves as well as the rms of the residuals. Figure 2 shows the obtained periodograms. To reduce the amount of graphical data we omitted all local minima until we got fewer than 3000 frequency points per diagram.

We started with the residuals of orbital solution I and found a first period near to $P_{1}=69 \mathrm{~d}$ (Fig. 2a). From this point, including the first additional period into our model, we get stable solutions from our new data alone and do not need to include the older data of lesser accuracy. At any point where we found 


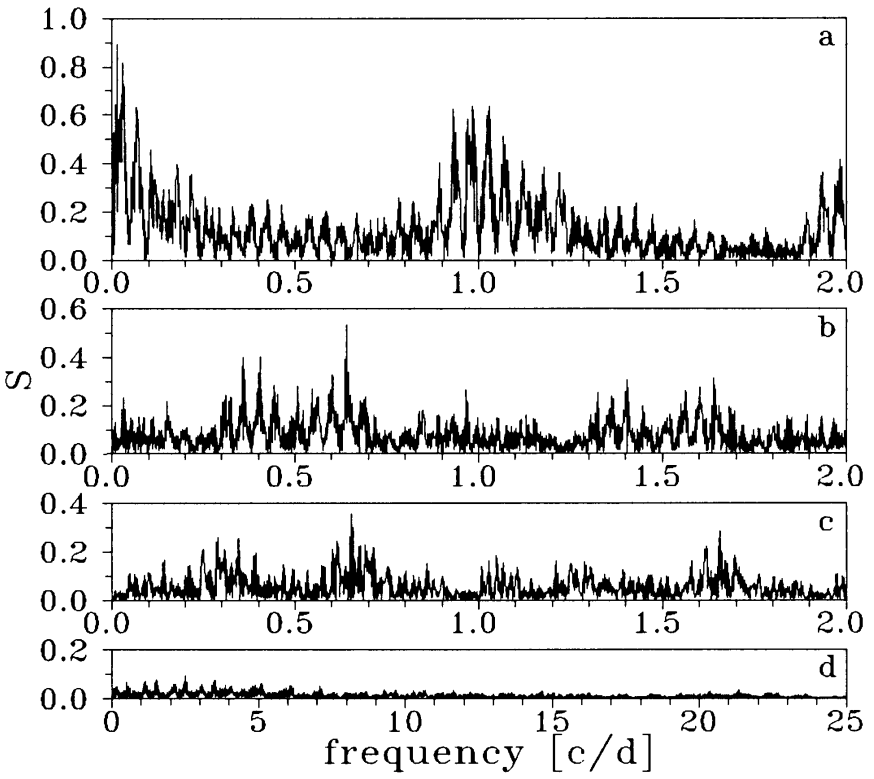

Fig. 2. Periodograms obtained at several steps of successive prewhitening, after subtracting orbital solution I (a)), and after subtracting also $P_{1}$ to $P_{3}(\mathbf{b})$ to d)). Periods $P_{1}$ to $P_{3}$ are the periods listed in Table 3.

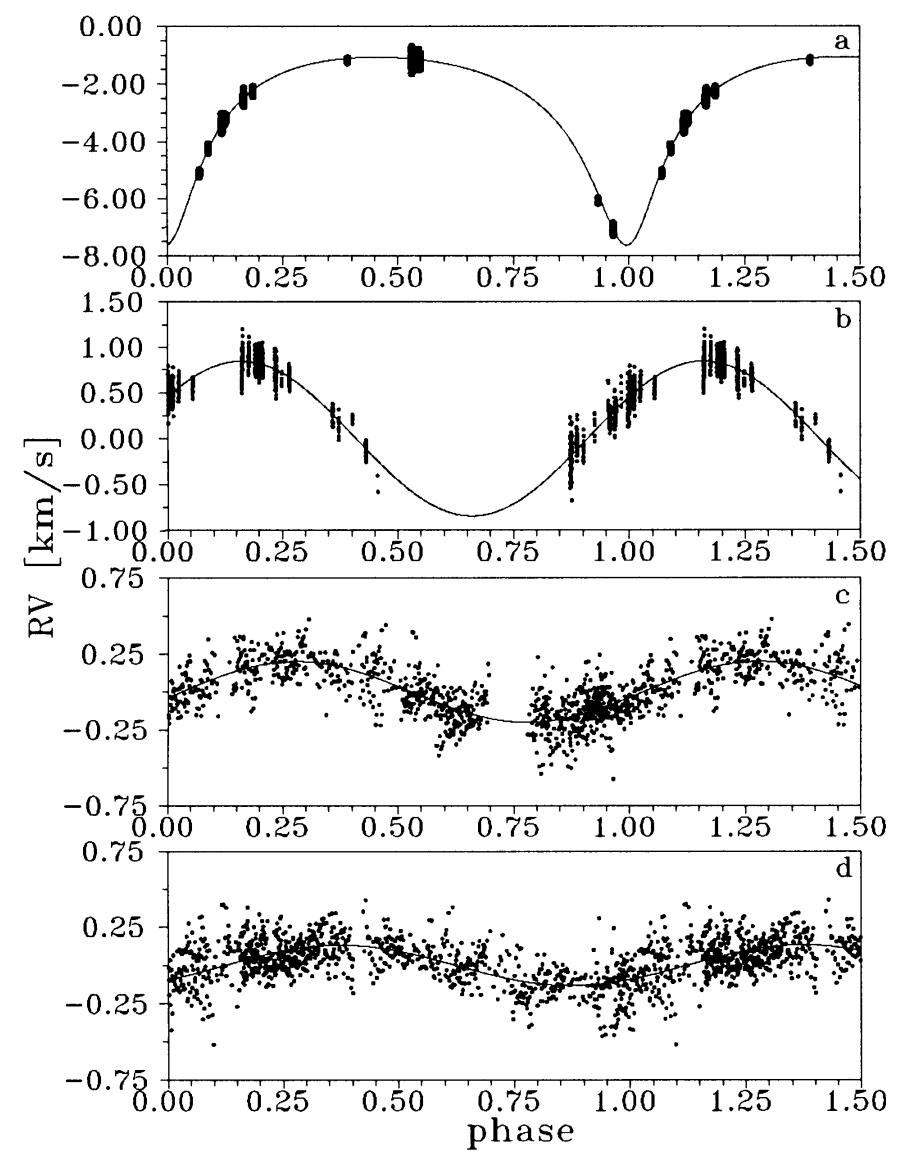

Fig. 3. Phase diagrams. a) Final cleaned orbital solution derived from the TLS (CCD) RVs and folded with the orbital period of 1306.82 . Panels b) to d) show the single contributions of the multiple frequency fit after subtracting all other contributions, folded with the periods of $69.232(\mathbf{b})), 1.56024(\mathbf{c}))$, and $1.53080(\mathbf{d})$ ).
Table 3. Results of period search. We list the periods and the corresponding half-amplitudes in the same order as they were found in the data, the orbital elements derived from the optimized solution (errors of the last two digits are in parentheses), and the rms of the residuals.

\begin{tabular}{lcrrl}
\hline \hline period [d] & $K\left[\mathrm{~m} \mathrm{~s}^{-1}\right]$ & \multicolumn{3}{c}{ orbital elements } \\
\hline$P_{1}=69.232$ & 842 & $P$ & $1306.82(96)$ & $\mathrm{d}$ \\
$P_{2}=1.56024$ & 201 & $K$ & $3.281(44)$ & $\mathrm{km} \mathrm{s}^{-1}$ \\
$P_{3}=1.53080$ & 131 & $e$ & $0.497(10)$ & \\
& & $T$ & $2450962.0(2.0)$ & \\
& & $\omega$ & $186.30(79)$ & $\mathrm{deg}$ \\
$\mathrm{rms}=122 \mathrm{~m} \mathrm{~s}^{-1}$ & & $\gamma$ & $-2.73(11)$ & $\mathrm{km} \mathrm{s}^{-1}$ \\
\hline
\end{tabular}

a new period, all previously determined periods were again optimized. In the next steps (Figs. 2b and c) we found the periods $P_{2}$ and $P_{3}$.

After including these three periods, the periodogram of the residuals (Fig. 2d) looks very smooth. The largest peak occurs at $0.39826 \mathrm{~d}$. Since the amplitude of this contribution of $52 \mathrm{~m} \mathrm{~s}^{-1}$ is distinctly below the estimated accuracy of our RVs and because this period is almost the mean time span of our observational runs, we do not regard this period as being real. Table 3 lists the final results.

\section{Stellar parameters of 2 Lyn}

From a line profile analysis of the averaged mean spectrum obtained from all RV-shifted CCD spectra (which was also used as the template for the RV-determination, see Sect. 2) we determined the projected rotational velocity to be $v \sin i=$ $(44 \pm 2) \mathrm{km} \mathrm{s}^{-1}$. This is exactly the same value as obtained by C\&A from their $2.4 \AA \mathrm{mm}^{-1}$ Reticon spectra of $S / N=200$ from lines near $4481 \AA$.

Assuming the parallax $\pi$ of the star as $21 . " 88$ (CDS data base), the effective temperature as $T_{\text {eff }}=9310 \mathrm{~K}$ (Table 5 of this paper) and a bolometric correction of -0.24 (Schmidt-Kaler 1981), the absolute bolometric magnitude of 2 Lyn is $M_{\text {bol }}=0.93$. To determine the radius and the mass of the star we use the radiation law and the mass-luminosity relation according to

$\log R / R_{\odot}=8.4-2 \log T_{\text {eff }}-0.2 M_{\text {bol }}$

and

$\log M / M_{\odot}=0.46-0.10 M_{\mathrm{bol}}$.

With $M_{\text {bol }}$ in mag and $T_{\text {eff }}$ in $\mathrm{K}$ we get for the radius and the mass the values $2.2 R_{\odot}$ and $2.3 M_{\odot}$, respectively.

For an oblique rotator, the equatorial rotational velocity $v$, the rotation period $P_{\text {rot }}$, and the stellar radius $R$ are related by

$v=50.61 R / P_{\text {rot }}$

if $R, v$, and $P_{\text {rot }}$ are expressed in solar radii, $\mathrm{km} \mathrm{s}^{-1}$, and days, respectively. With $v \sin i=44 \mathrm{~km} \mathrm{~s}^{-1}$ the rotational period of the primary should be shorter than about 2.5 days. If we interprete the periods $P_{2}$ or $P_{3}$ from Table 3 as the rotation period of $2 \mathrm{Lyn}$, the aspect angle $i$, which is the inclination between the rotation axis and the line of sight, would be about $37^{\circ}$. 
Table 4. Ions, multiplet numbers, wavelengths (in $\AA$ ), oscillator strengths, and equivalent widths $W_{1}$ of HD 169981 and $W_{2}$ of 2 Lyn (in mÅ).

\begin{tabular}{|c|c|c|c|c|c|c|c|c|c|c|c|c|c|c|c|c|c|}
\hline ion & RMT & $\lambda$ & $\log g f$ & $W_{1}$ & $W_{2}$ & ion & RMT & $\lambda$ & $\log g f$ & $W_{1}$ & $W_{2}$ & ion & RMT & $\lambda$ & $\log g f$ & $W_{1}$ & $W_{2}$ \\
\hline \multirow[t]{4}{*}{$\mathrm{CI}$} & 6 & 4771.75 & -1.69 & & 42 & $\mathrm{Cr}$ II & 43 & 5313.59 & -1.65 & 50 & 29 & $\mathrm{Fe} \mathrm{I}$ & 168 & 6393.61 & -1.43 & 22 & \\
\hline & 13 & 4932.05 & -1.78 & & 23 & & 43 & 5308.44 & -1.85 & 32 & & & 816 & 6400.01 & -0.29 & 37 & \\
\hline & 12 & 5052.12 & -1.65 & 30 & 45 & & 43 & 5310.70 & -2.28 & 18 & & $\mathrm{Fe} \mathrm{II}$ & 38 & 4508.28 & -2.25 & & 101 \\
\hline & 11 & 5380.24 & -1.84 & 14 & & & 43 & 5334.88 & -1.56 & 52 & 24 & & 37 & 4515.34 & -2.45 & & 84 \\
\hline \multirow[t]{2}{*}{ O I } & 10 & 6156.78 & -0.69 & 29 & & & 23 & 5407.62 & -2.15 & 20 & & & 37 & 4520.22 & -2.60 & & 73 \\
\hline & 10 & 6158.18 & -0.31 & & 77 & & 23 & 5420.90 & -2.46 & 19 & & & 38 & 4522.63 & -2.03 & & 106 \\
\hline \multirow[t]{2}{*}{$\mathrm{Mg} \mathrm{I}$} & 11 & 4703.00 & -0.67 & & 76 & & 50 & 5502.05 & -2.00 & 24 & & & 38 & 4576.33 & -2.97 & & 54 \\
\hline & 9 & 5528.40 & -0.62 & 73 & 67 & & 50 & 5508.60 & -2.11 & 20 & & & 38 & 4620.51 & -3.24 & & 43 \\
\hline Si II & 2 & 6371.36 & -0.16 & 146 & & $\mathrm{Fe} I$ & 41 & 4404.75 & -0.14 & & 91 & & 37 & 4629.34 & -2.33 & & 91 \\
\hline \multirow[t]{11}{*}{ Ti II } & 105 & 4163.65 & -0.21 & & 76 & & 350 & 4466.55 & -0.60 & & 33 & & 186 & 4635.33 & -1.65 & & 32 \\
\hline & 40 & 4464.45 & -1.81 & & 55 & & 554 & 4736.78 & -0.71 & 38 & & & 43 & 4731.45 & -3.00 & & 48 \\
\hline & 31 & 4501.27 & -0.76 & & 117 & & 687 & 4966.10 & -0.87 & 26 & & & 36 & 4993.36 & -3.64 & 50 & 25 \\
\hline & 82 & 4571.97 & -0.23 & & 128 & & 1146 & 5364.87 & +0.23 & 43 & 25 & & 49 & 5197.56 & -2.11 & & 86 \\
\hline & 92 & 4779.99 & -1.32 & 52 & & & 1146 & 5367.47 & +0.44 & 53 & 26 & & 49 & 5234.62 & -2.23 & & 92 \\
\hline & 92 & 4805.11 & -0.96 & 75 & & & 1146 & 5369.97 & +0.54 & 66 & & & 49 & 5254.93 & -3.23 & & 32 \\
\hline & 86 & 5185.90 & -1.37 & 47 & & & 15 & 5371.49 & -1.65 & & 33 & & 41 & 5284.09 & -2.99 & 86 & \\
\hline & 70 & 5188.69 & -1.05 & & 76 & & 1146 & 5383.37 & +0.65 & & 47 & & 48 & 5362.87 & -2.74 & & 71 \\
\hline & 103 & 5211.54 & -1.36 & 22 & & & 1165 & 5410.91 & +0.40 & 49 & 36 & & & 5387.06 & +0.52 & & 13 \\
\hline & 69 & 5336.81 & -1.63 & 50 & & & 686 & 5569.63 & -0.49 & 33 & & & 48 & 5414.09 & -3.54 & 39 & \\
\hline & 114 & 4911.21 & -0.65 & 42 & & & 686 & 5586.75 & -0.12 & & 37 & & 49 & 5425.27 & -3.16 & 55 & \\
\hline \multirow[t]{7}{*}{ Cr II } & 44 & 4558.66 & -0.45 & & 92 & & 686 & 5615.65 & +0.05 & 70 & 43 & & 55 & 5534.83 & -2.73 & & 57 \\
\hline & 44 & 4588.22 & -0.63 & & 81 & & 207 & 6065.49 & -1.53 & 17 & & & 46 & 6084.11 & -3.78 & 30 & \\
\hline & 44 & 4592.07 & -1.22 & & 45 & & 169 & 6136.62 & -1.40 & 27 & & & 74 & 6238.38 & -2.63 & 70 & \\
\hline & 44 & 4616.64 & -1.36 & & 40 & & 169 & 6191.56 & -1.42 & 20 & & & 74 & 6416.91 & -2.65 & 57 & \\
\hline & 44 & 4634.10 & -0.99 & & 60 & & 207 & 6230.73 & -1.28 & 26 & & & 40 & 6432.65 & -3.52 & 51 & \\
\hline & 30 & 4812.35 & -1.73 & 36 & & & 816 & 6246.33 & -0.73 & 17 & & & & & & & \\
\hline & 43 & 5237.35 & -1.16 & & 53 & & 169 & 6252.56 & -1.69 & 18 & & & & & & & \\
\hline
\end{tabular}

The minimal mass of the secondary can be estimated from the mass function (note that here the angle $i$ is the orbital inclination)

$$
\frac{M_{2}^{3} \sin ^{3} i}{\left(M_{1}+M_{2}\right)^{2}}=1.036 \times 10^{-7} K_{1}^{3} P_{\text {orb }}\left(1-e^{2}\right)^{3 / 2} \text {. }
$$

Using the orbital elements from Table 3 and the mass of the primary of $2.3 M_{\odot}$, the mass of the secondary has to be larger than $0.27 M_{\odot}$. Normally, for asynchronous and eccentric systems as in our case - the rotational axes are randomly inclined to their orbital planes. In the special case of coplanarity we would get from the abovederived aspect angle of $37^{\circ}$ (and interpreting one of the periods $P_{2}$ or $P_{3}$ as the rotation period) a mass of the secondary of $0.46 M_{\odot}$.

\section{Elemental abundances}

\subsection{Atmospheric parameters}

The effective temperatures of HD 169981 and 2 Lyn have been estimated from the Strömgren photometry using Napiwotski's et al. (1993) code. The microturbulent velocity was derived supposing that there is no dependence between the iron abundance of the Fe I lines (they are the most numerous ones in the available part of the spectrum of both stars) and their equivalent widths (EWs). By keeping the condition of ionizational balance, i.e. equality of the iron abundance following from Fe I and Fe II lines, we determined then the surface gravity. The WIDTH9 code and Kurucz's (1995) ATLAS9 atmospheres have been used to derive the elemental abundances. An appropriate model atmosphere was interpolated from the Kurucz grid, oscillator strengths were taken from Reader et al. (1980) for C I, Biemont et al. (1991) for O I, and from the VALD database for all other elements. EWs were measured in Gaussian approximation and analysed by means of the WIDTH9 code.

The spectroscopic data of the analysed lines of both stars are given in Table 4. For the atmospheric parameters we obtained the values listed in Table 5. Errors correspond to $1 \sigma$. Comparing our values with the atmospheric parameters of 2 Lyn given by C\&A, $T_{\text {eff }}=9295 \mathrm{~K}, \log g=4.1$, and $v_{\mathrm{t}}=2.1 \mathrm{~km} \mathrm{~s}^{-1}$ we notice a complete agreement within the given errors.

\subsection{Atmospheric abundances}

Although HD 169981 is known to be a binary, we performed the spectroscopic analysis of the star as a single one. This 
Table 5. Atmospheric parameters of HD 169981 and 2 Lyn.

\begin{tabular}{lccc}
\hline \hline star & $\begin{array}{c}T_{\text {eff }} \\
(\mathrm{K})\end{array}$ & $\begin{array}{c}\log g \\
(\mathrm{CGS})\end{array}$ & $\begin{array}{c}v_{\mathrm{t}} \\
\left(\mathrm{km} \mathrm{s}^{-1}\right)\end{array}$ \\
\hline HD 169981 & $8700 \pm 100$ & $3.3 \pm 0.1$ & $2.05 \pm 0.1$ \\
2 Lyn & $9310 \pm 100$ & $4.1 \pm 0.1$ & $2.00 \pm 0.1$ \\
\hline
\end{tabular}

assumption is justified because the binary consists of an A-type primary and a K-type secondary having a significant difference in luminosity of about 6 mag (Lehmann et al. 2001).

The derived relative-to-solar elemental abundances of HD 169981 and 2 Lyn are given in Table 6. It contains also the abundances of the sun and those ones derived for 2 Lyn by C\&A. For comparison the abundances following from Fe I and Fe II are again separately listed. All abundances derived for 2 Lyn agree within the listed errors with the abundances obtained by C\&A. Nevertheless, our abundances are, on average, slightly larger than C\&A's values. To look for possible reasons we correlated our $\log g f$ values as well as the derived EWs with the values of Table 5 in C\&A. Due to the different spectral regions there is only a small number of lines that were used both by C\&A and us. From these 22 lines ( 9 Fe II, 3 Fe I, 5 Cr II, 4 Ti II, $1 \mathrm{Mg}$ I) we find, applying a 2- $\sigma$ clipping,

$\frac{g f_{1}}{g f_{2}}=1.00 \pm 0.18$

$\frac{E W_{1}}{E W_{2}}=1.21 \pm 0.56$

where index 1 refers to our values and index 2 to those of C\&A. There are no significant differences between the chosen $\log g f$ values. The situation regarding the EWs is the same as for the derived abundances: the values agree within $1 \sigma$, on average our values are larger by a factor of 1.2 , however.

There may be two reasons for the small difference in abundances. First there could be differences in how scattered light was removed from the high-resolution spectra. Secondly, the small difference in abundances may be due to the fact that C\&A used a fit of artificially broadened profiles to derive the EWs whereas we used only a simple fit of Gaussians. Because of the excellent agreement in the derived value of $v \sin i$ we assume the second cause to be responsible.

\section{Discussion}

The investigation of the RVs of 2 Lyn shows the star to be a long-period spectroscopic binary. Independent of our assumptions on the existence of further short-term periodicity, we can derive an orbital period of about 3.6 years. The analysis of a data set comprising our newly obtained RVs and older measurements shows that the derived orbital solution strongly depends on the applied weighting scheme. Because of the poor phase coverage, the derived RV half-amplitude varies according to the given weights between 3 and $9 \mathrm{~km} \mathrm{~s}^{-1}$ and the eccentricity between 0.53 and 0.78 .

In order to get a stable solution and the most accurate orbital elements we use only our new high-accuracy data
Table 6. Mean abundances $\log (\mathrm{El} / \mathrm{H})$ of HD 169981 and 2 Lyn.

\begin{tabular}{lcccc}
\hline \hline Ion & $\begin{array}{c}\mathrm{El} / \mathrm{H} \\
\mathrm{HD} \mathrm{169981}\end{array}$ & $\begin{array}{c}\mathrm{El} / \mathrm{H} \\
2 \mathrm{Lyn}\end{array}$ & $\begin{array}{c}\mathrm{El} / \mathrm{H} \\
(\mathrm{C} \& \mathrm{~A})^{1}\end{array}$ & $\begin{array}{c}\mathrm{El} / \mathrm{H} \\
\mathrm{Sun}^{2}\end{array}$ \\
\hline $\mathrm{C} \mathrm{I}$ & $-3.84 \pm 0.13$ & $-3.45 \pm 0.12$ & $-3.54 \pm 0.16(2)$ & -3.45 \\
O I & $-3.44 \pm 0.00$ & $-3.19 \pm 0.00$ & & -3.13 \\
Mg I & $-4.53 \pm 0.00$ & $-4.39 \pm 0.03$ & $-4.51 \pm 0.21(6)$ & -4.42 \\
Si II & $-4.48 \pm 0.00$ & & & -4.45 \\
Ti II & $-7.18 \pm 0.08$ & $-6.76 \pm 0.17$ & $-6.90 \pm 0.26(34)$ & -6.98 \\
Cr II & $-6.32 \pm 0.10$ & $-6.34 \pm 0.06$ & $-6.39 \pm 0.18(22)$ & -6.33 \\
Fe I & $-4.44 \pm 0.07$ & $-4.55 \pm 0.07$ & $-4.68 \pm 0.17(68)$ & -4.50 \\
Fe II & $-4.42 \pm 0.07$ & $-4.54 \pm 0.10$ & $-4.70 \pm 0.18(39)$ & -4.50 \\
\hline
\end{tabular}

${ }^{1}$ Abundances of 2 Lyn derived by Caliskan \& Adelman (1997). The numbers of used lines are given in parentheses.

${ }^{2}$ Solar abundances are those recommended by Grevesse et al. (1996).

and include additional sinusoidal variations. In doing this we find three additional periodic variations with periods of $P_{1}=$ $69.232 \mathrm{~d}, P_{2}=1.56024 \mathrm{~d}$, and $P_{3}=1.53080 \mathrm{~d}$.

Although the phase diagram of the $P_{1}$ period (Fig. 3b) has a larger gap, we consider this period as reliable. The reason is twofold. First, the amplitude of this contribution of $0.84 \mathrm{~km} \mathrm{~s}^{-1}$ is far above the errors of calculation and we find no other period which gives a comparably good fit. Secondly, the period is, compared to the time sampling of our data, sufficiently long so that the occurrence of the gap in the phase diagram can be explained by chance. However, at present we have no definite explanation for the existence of $P_{1}$; perhaps the orbital motion of a third body is the cause.

From our estimation of the stellar parameters of $2 \mathrm{Lyn}$ we favour the second period found, $P_{2}=1.56$, as the rotation period of the star. The occurrence of the very near doublet $\left(P_{2}, P_{3}\right)$, however, whose separation corresponds to a period of $81 \mathrm{~d}$ and being on the order of $P_{1}$, could be an artifact from the applied mathematical procedure. Only further spectroscopic observations will help to clarify the reality and nature of the indicated periods.

The derivation of the elemental abundances of 2 Lyn mainly has been carried out to check our result with that of the comprehensive study made by C\&A. We find sufficient agreement between the abundances studied and suggest the star is a normal main sequence star of spectral type A2.

Concerning HD 169981 the derived value of the surface gravity of $\log g=3.3$ favours the primary to be an early Atype giant as postulated by Lehmann et al. (2001) in their binary model. Most of the investigated elements have solar-like abundances and only carbon and oxygen show some slight underabundance.

Acknowledgements. The authors are indebted to the referee, Dr. Adelman, for his useful comments and the careful proofreading of the manuscript which helped us to improve the paper.

\section{References}

Abt, H. A., \& Morrell, N. I. 1995, ApJS, 99, 135

Antonello, E., Arienti, F., Fracissini, M., et al. 1978, A\&A, 66, 37 
Biemont, E., Hibbert, A., Godefoid, M., Vaeck, N., \& Fawcett, B. C. 1991, ApJ, 375, 818

Caliskan, H., \& Adelman, S. J. 1997, MNRAS, 288, 501

Grevesse, N., Noels, A., \& Sauval, J. 1996, ASP. Conf. Ser., 99, 117

Kurucz, R. L. 1995, ASP Conf. Ser., 78, 205

Lehmann, H., Hildebrandt, G., Panov, K. P., \& Scholz, G. 2001, A\&A, 373, 960 (Paper I)

Napiwotski, R., Schönberner, D., \& Wenske, V. 1993, A\&A, 268, 653

Reader, J., Corliss, C., Wiese, W. L., \& Martin, G. A. 1980, National Standard Reference Ser., Wahington, National Bureau of Standards

Schlesinger, F. 1908, Publ. Allegheny Obs., 1, 35
Schmidt-Kaler, Th. 1981, Physical parameters of the stars, in LandoltBörnstein. Zahlenwerte und Funktionen aus Naturwissenschaften und Technik, Neue Serie, Gruppe IV: Astronomie, Astrophysik und Weltraumforschung; Band 2b (Berlin, Heidelberg, New York: Springer-Verlag), 455

Scholz, G., Lehmann, H., Hildebrandt, G., et al. 1998, A\&A, 337, 447 (Paper II)

Solano, E., \& Fernley, J. 1997, A\&AS, 122, 131

Uesugi, A., \& Fukuda, J. 1982, Catalogue of Stellar Rotational Velocities, rev. ed., University of Kyoto, Department of Astronomy

Young, R. K. 1919, Pub. Dom. Astrophys. Obs., 1, 131 